\title{
Response to Evaluation of coronary artery variations using dual-source coronary computed tomography angiography in neonates with transposition of the great arteries
}

\author{
M. Kardos ${ }^{1}$
}

Received: 19 June 2019 / Accepted: 11 July 2019 / Published online: 24 July 2019

(c) Japan Radiological Society 2019

To the Editor,

I am writing with regard to the article titled, "Evaluation of coronary artery variations using dual-source coronary computed tomography angiography in neonates with transposition of the great arteries" published in April 2019 issue of JJR [1].

I believe that evaluation of coronary arteries in patient with transposition of the great arteries is crucial for the future open heart surgery.

The authors of this paper tried to compare these two modalities in evaluation of coronary arteries, but result was already known at the beginning. A surgeon needs to know especially proximal anatomy of coronary arteries before the surgery. An experienced physician, performing echo, provides enough information for the surgeon. CT angiography provides better spatial resolution than echo. It should be reserved just for cases when echocardiography (echo) is not helpful. Using the breath-hold and retrospective ecg imaging is also questionable. For the evaluation of the proximal parts of coronary arteries the breath-hold is not necessary and significantly prolongs the entire examination. The proximal coronary artery course is most critical and higher heart rates are tolerated. Scanners with the best temporal resolution are most likely to achieve diagnostic images in patients with the highest heart rates. Consider medication to lower heart rate for coronary imaging is possible [2]. The very important issue is dose-reduction especially in newborns. The author thinks that prospective ecg imaging of coronaries is also possible and this type of

M. Kardos

kardi.marek@gmail.com

1 Department of Functional Diagnostics, Children's Cardiac

Center, Limbova 1, 83351 Bratislava, Slovakia acquisition reduces dose more significantly than retrospective ecg imaging.

Sincerely,

M. Kardos

Funding The author(s) received no financial support for the research, authorship, and/or publication of this article.

\section{Compliance with ethical standards}

Conflict of interest The author(s) declared no potential conflicts of interest with respect to the research, authorship, and/or publication of this article.

Ethical statement All applicable institutional and/or national guidelines for the care and use of animals were followed.

\section{References}

1. Odawara Y, Kawamura N, Yamasaki Y, et al. Evaluation of coronary artery variations using dual-source coronary computed tomography angiography in neonates with transposition of the great arteries. Jpn J Radiol. 2019;37:308-14.

2. Han BK, Rigsby CK, Leipsic J, Bardo D, Abbara S, Ghoshhajra $\mathrm{B}$, et al. Computed tomography imaging in patients with congenital heart disease, Part 2: technical recommendations: an expert consensus document of the Society of Cardiovascular Computed Tomography (SCCT) Endorsed by the Society of Pediatric Radiology (SPR) and the North American Society of Cardiac Imaging (NASCI). JCCT. 2015;9:493-513.

Publisher's Note Springer Nature remains neutral with regard to jurisdictional claims in published maps and institutional affiliations. 